\title{
Suicide, unemployment, and domestic gas detoxification in Britain
}

\author{
N KREITMAN AND S PLATT
}

From the MRC Unit for Epidemiological Studies in Psychiatry, University Department of Psychiatry, Royal Edinburgh Hospital, Edinburgh EH10 5HF, UK

SUMmARY Between 1962 and 1971 the United Kingdom suicide rate fell while the unemployment rate rose, contrary both to earlier British experience and recent trends in other developed countries. It is hypothesised that the "British anomaly" is due to the coincident detoxification of domestic gas after 1963. Unemployment and suicide by means other than domestic gas were found to correlate +0.88 between 1955 and 1980 , and +0.70 between 1964 and 1974 . These findings are discussed with respect to the role of availability of method in the epidemiology of suicide. It is also suggested that the relation between unemployment and suicide is complex and probably largely indirect.

This paper reports a further study on the effects of the detoxification of domestic gas on the suicide rate in Britain and presents an additional analysis using unemployment data which may help to resolve some of the remaining controversy surrounding this topic. Since death by inhalation of domestic gas is almost entirely due to carbon monoxide poisoning such deaths will usually be described below as "CO deaths."

It may help the.reader to recall that in Britain the detoxification of domestic gas was begun in 1957 when a "typical" supply contained $14 \%$ of $\mathrm{CO}$. Initially progress was slow and by 1968 the corresponding figure was $12 \%$, but thereafter a rapid fall ocurred so that by 1972 the proportion was below $1 \% .^{1}$

\section{Background}

The use of different methods of suicide in the United Kingdom since the last century has been analysed by Farmer. ${ }^{2}$ After grouping closely similar methods, such as poisonings by different drugs, he concluded that the rates for the main groups varied independently, with no evidence of compensatory shifts. He also commented that the introduction of domestic gas for heating and cooking between 1900 and 1920 was associated with the emergence of $\mathrm{CO}$ suicide with no compensatory decrease in suicide due to other methods, and that similarly the detoxification of domestic gas in the 1960s had led to a fall in the former group but no corresponding increase in other types of suicide.
Hassall and Trethowan commented on the rapid fall in the suicide rate in the Birmingham area between 1963 and 1970, a period during which the CO content of the local gas supplies fell from about $20 \%$ to $2 \cdot 5 \% .^{3}$ They showed that the decrease resulted from the dramatic reduction of CO-suicide, whereas suicide by other methods showed no change. Subsequently Kreitman ${ }^{1}$ summarised the debate that followed the Hassall and Trethowan study, and analysed data from England and Wales, and separately for Scotland, concerning suicide by domestic gas and by other methods within each agesex subgroup of the population over the period 1960 to 1971 . Figure 1 is a representative finding from that study. No evidence was found that the changes were artefacts due to the introduction of the revised International Classification of Disease in 1971 or to changes over time in the allocation of deaths between suicide and accidental death categories. The hypothesis that the decline in the total suicide rate could be directly ascribed to the reduced availability. of toxic domestic gas was explored by examining the implications of this thesis for changes in the pattern of parasuicide during the same period; the role of changes in primary health care was also considered. The conclusion drawn was that the hypothesis was probably supported, and that individuals in a presuicidal state who did not have access to a preferred method of death did not to any substantial degree adopt alternative strategies for ending their lives.

The conclusion has major implications for the understanding of variations in suicide rates and as a 
pointer to possible preventive action. It entails the rejection of an alternative hypothesis that might be advanced for changes of the type illustrated in fig 1 . It could be argued that during the period between 1963 and 1971 the suicide rate was declining for extraneous reasons; at the same time the progressive detoxification of domestic gas meant that by the end of the period it was almost impossible for anyone to kill themselves by this method. These two factors chanced to coincide, producing the curves illustrated in fig 1. Such a hypothesis implies a nearly exact equilibrium between the postulated (and unexplained) primary decline in the suicide rates and the decreasing toxicity of domestic gas; these would have to be so combined as to leave the non-CO suicide rate unchanged or slightly increasing. A balance of this sort might be judged improbable but clearly the hypothesis cannot be rejected out of hand.

Further evaluation of the two explanations requires other sources of data. Sainsbury (personal communication) reported having studied pairs of cities or regions in the United Kingdom which were selected such that each member of the pair had an approximately equal suicide rate at the beginning of the period but where the detoxification of gas proceeded rapidly in one but slowly or not at all in the other. At the end of the study period there was still no difference between the total suicide rates in the two groups. These results, however, have not been published. A second source of data concerns suicide in the different provinces of the Netherlands, the only country in Europe apart from the United Kingdom to have had an appreciable proportion of CO-suicides. Various provinces in the Netherlands underwent gas detoxification at different times. A recent WHO report comments that such changes were not reflected in parallel decreases in the total suicide rate, but again no data are given. ${ }^{4}$ Thus neither of these important studies can be evaluated at present.

The remainder of this paper will be concerned with evidence of another, more indirect sort. Sainsbury et al have given a preliminary report of an analysis of trends in suicide between 1961-3 and 1971-3 in 18 European countries ${ }^{5}$ : suicide had increased in 15 countries and fallen in three. For these years the authors assembled data for a selection of 15 social variables and for sex specific suicide rates. Their most relevant analysis for present purposes concerned changes in the social variables over the study period in relation to changes in the suicide rates. They found that for men the changes on seven social variables correlated significantly with the change in suicide rate, and of these the strongest were increase in TV set home ownership $(r=0.61)$ and $\frac{9}{D}$ 을 unemployment $(r=0 \cdot 36)$. For women five $\stackrel{-}{-}$ significant variables emerged of which the two most powerful were the same as for the men $(r=0.42$ and 0.33 respectively).

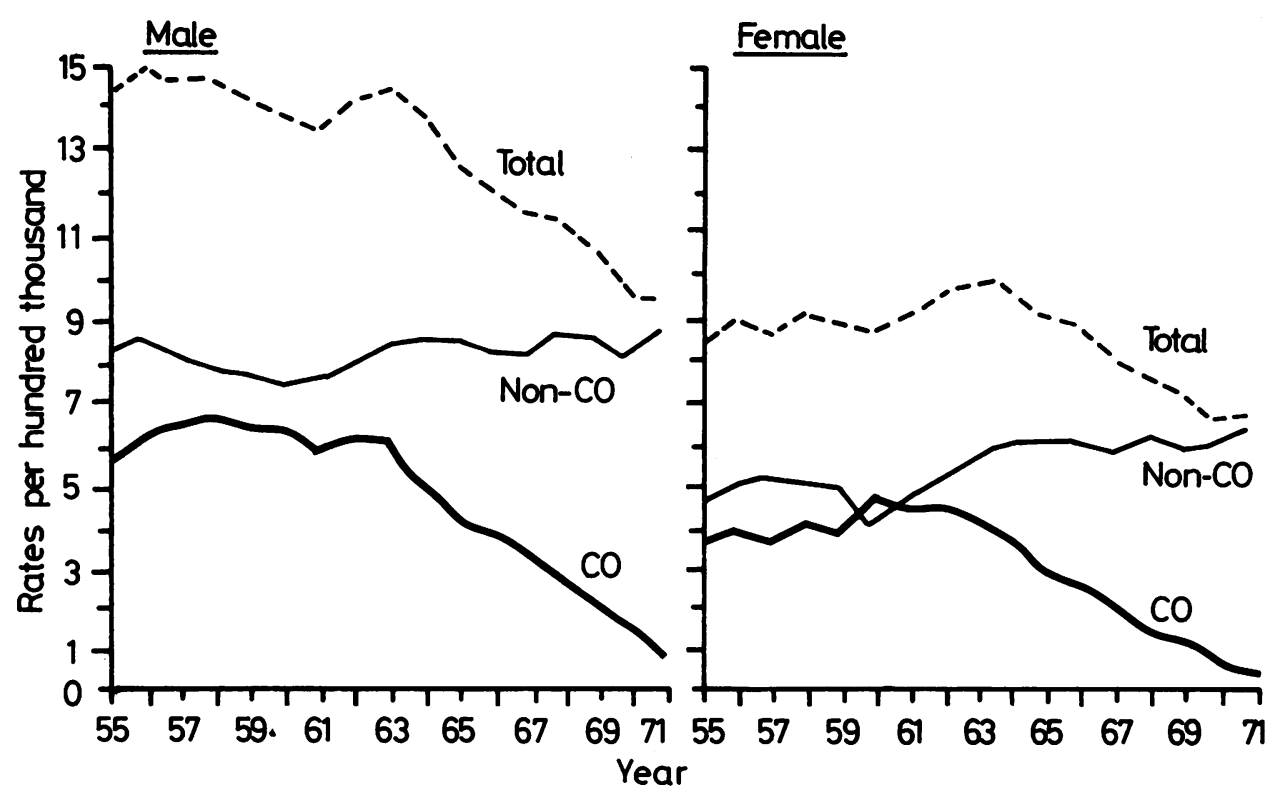

Fig 1 England and Wales: sex specific suicide rates by mode of death. 
The authors then divided the 18 countries into two groups according to the magnitude of change in their suicide rates, and carried out a discriminant function analysis using the change scores on the whole array of variables. The formula showed change in marital status rates as the most powerful discriminator, followed by unemployment. Although in general the discriminant function formula performed well, on closer testing a poor fit was obtained for England and Wales. The formula predicted that England and Wales would come seventh in rank among the 18 countries, whereas in fact it came first, being the country showing the largest decrease in suicide.

Given the important role of unemployment, the result is strange, since in Britain unemployment rose by approximately 50\% between 1961 and 1971 (see below) yet the suicide rate fell by $34 \%$. This anomaly prompted us to re-examine the relation between suicide and unemployment. Platt has recently summarised the many publications on this topic. ${ }^{15}$ An illustrative study particularly relevant to the present issue is that by Boor ${ }^{6}$ whose findings are summarised in table 1 . Boor used an index of unemployment and suicide rate in both of which men and women were combined. For the years between 1962 and 1976 he calculated, using WHO sources, the correlations between unemployment and suicide in eight industrialised countries. The table shows that six countries showed substantial positive correlations over the study period, which in four countries were statistically significant. Italy, which is probably the least industrialised of the nations in the list, showed no change. The United Kingdom showed a significant negative correlation.

It seems, then, that in relation to the other countries of Europe, as analysed by Sainsbury et al, ${ }^{5}$ or with respect to the group of developed countries examined by Boor, ${ }^{6}$ the United Kingdom is

Table 1 Correlations $\left(r_{s}\right)$ between rates for unemployment and suicide in eight countries (from Boor ${ }^{8}$ )

\begin{tabular}{lll}
\hline Country & Period & Correlation \\
\hline United States & $1962-76$ & $+0.79^{*}$ \\
France & $1962-74$ & $+0.64^{*}$ \\
Japan & $1962-76$ & $+0.53^{*}$ \\
Canada & $1962-75$ & $+0.51^{*}$ \\
Sweden & $1962-76$ & +0.40 \\
West Germany & $1962-75$ & +0.39 \\
Italy & $1962-74$ & 0.00 \\
Britain & & $-0.59^{*}$ \\
\hline
\end{tabular}

$* \mathrm{p}<0.05$. conspicuously anomalous over the period between the beginning of the 1960 s and the middle of the 1970 s. The paradox is further heightened by noting that at earlier times the association in the United Kingdom between suicide and unemployment was the same as that reported for so many other countries for recent years. Figure 2 is from Swinscow ${ }^{7}$ and illustrates numbers of suicides and numbers of unemployed men between 1923 and 1947. The two variables correlate $r=0.93(p<0.01)$. The corresponding correlation for female suicide and female unemployment was much lower $(r=0 \cdot 47, \mathrm{~ns})$ but for women unemployment data were and remain highly unsatisfactory.

In summary, British experience since 1960 is anomalous with respect to $(a)$ the rest of Europe between 1961 and 1971, (b) a number of other developed countries over a roughly comparable period, and $(c)$ the changes in Britain itself in earlier years.

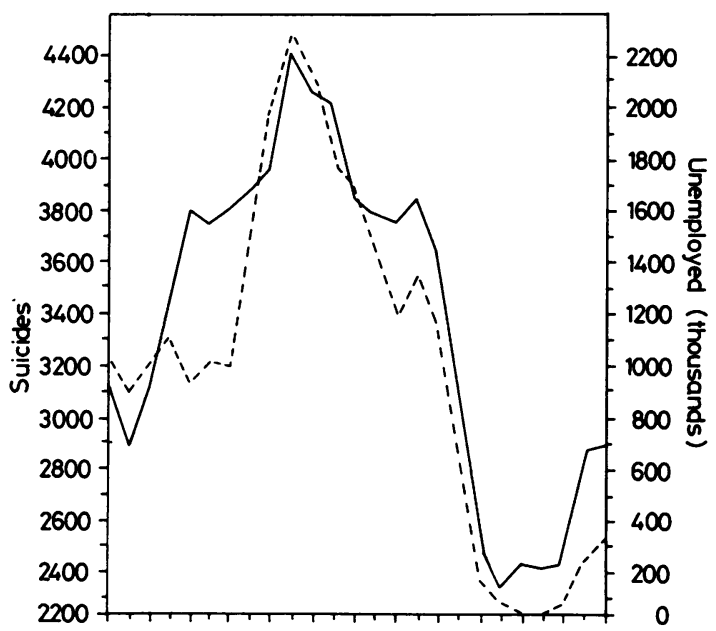

Fig 2 Comparisons between numbers of male suicides $(-(-)$, and numbers of unemployed men (- - ) during 1923-47 (Britain).

\section{Hypothesis}

We hypothesise that the paradox can be explained, at least for men, by a specific confounding effect on the correlation between unemployment and the suicide rate between 1963 and 1971 (or 1972), an effect represented by a true decrease in the suicide rate attributable to the detoxification of domestic gas. The hypothesis implies that the $\mathrm{CO}$ and non-CO components of the total suicide rate have varied independently, the former in parallel with detoxification whereas the latter has maintained the 
traditional correlation with unemployment. The prediction to be tested is that the unemployment rate, though negatively correlated with the total suicide rate, would be positively correlated with suicide due to non-CO modes of death.

A secondary hypothesis distinguishing the 196371 period from earlier and subsequent decades was subsequently investigated and tested, as will be explained below.

\section{Method}

A preliminary test along the lines of Boor's analysis ${ }^{6}$ supported the primary hypothesis, and we were thereby encouraged to look at more precise data.

We needed to define both unemployment rates and suicide rates. The unemployment rate referred to wholly unemployed individuals, including school leavers, on the register of local employment offices and careers offices. The numerator of the rate for each year was based on the average of 12 estimates each relating to the numbers of unemployed on a given day in successive months, as published in the Employment Gazette and its forerunners. From 1967 onwards adult students were excluded from the statistics but the numbers were very small. Note that the major shift from a registrant to a claimant basis for the derivation of unemployment statistics occurred after the study period. The denominator we adopted was the total number of men aged 15 and over as given in the Registrar General's mid-year estimates. This is a larger population base than that used by the Department of Employment (all employees in employment plus the registered unemployed) or than that commonly derived from the Decennial Census (all economically active). Since our denominators include those aged 65 and over, and the economically inactive, the resulting rates are correspondingly lower than those published by the Department of Employment: nevertheless, they correlate perfectly.

The suicide rates were calculated from the Registrar General's annual reports. They refer to the population aged 15 and over.

Data from England and Wales were pooled with those from Scotland*, so that all rates refer to Britain.

Pearson product-moment correlation coefficients were used throughout, but all calculations were repeated using non-parametric methods, with substantially the same results.

*There is some evidence that the Scottish data (which of course represent only a small proportion of that from Britain as a whole) show some interesting differences from that for England and Wales. These are not explored here.

\section{Results}

It seemed useful to begin by considering the whole period between 1955 and 1980. The data are illustrated in fig 3 and the correlations are given in table 2 . The table shows that for the period 1955-80 male unemployment rates had a substantial negative correlation with rates for suicides by all causes, but a strong positive correlation with rates for suicide by means other than domestic gas.

A similar reversal was found, from $r=-0.80$ to $r=0.66$, when male unemployment was correlated with female suicides, a point which will be considered in the discussion.

We also considered more closely, and as required by our hypothesis, the period between 1964 when domestic gas detoxification was started and 1972 when the process was completed. We predicted that during the preceeding period, from 1955 to 1963 , male unemployment would be positively related with male suicide rates for all causes and for non-CO causes; that, as already stated, for the period 1964 to 1972 there would be a negative correlation between male unemployment rates and male suicide by all methods but a positive correlation between the former and suicide by non-CO methods, while for the later period of 1973 onwards a positive correlation would again be found between male unemployment and male suicides by non- $\mathrm{CO}$ methods (domestic gas having by this time become virtually non-toxic and accounting for hardly any suicidal deaths). Columns 2,3 , and 4 of table 2 give the results. Our prediction is not supported for the years 1955 to 1963 . For the remaining two periods the results are as hypothesised, notwithstanding the low number of observations on which they are based.

Table 2 Correlations ( $r$ ) between male unemployment rates and male suicide rates, by method: Britain 1955-80

\begin{tabular}{lcccl}
\hline & $1955-80$ & $1955-63$ & $1964-72$ & $1973-80$ \\
\hline All methods & $-0.73^{* *}$ & 0.13 & $-0.89^{* *}$ & $0.60(\mathrm{p}=0.057)$ \\
Non-CO only & $0.88^{* *}$ & 0.06 & $0.70^{*}$ & $0.71^{*}$ \\
\hline
\end{tabular}

$*$ p $<0.05$ on two-tail test.

$* \mathrm{p}<0.01$ on two-tail test.

\section{Discussion}

The two sets of rates we have been considering are of different types. Those for unemployment are summary annual measures of the point prevalence of spells of unemployment, whereas the suicide rates are annual mortality rates. Further, for the unemployment rates we have deliberately used the total adult population, although individuals over the 


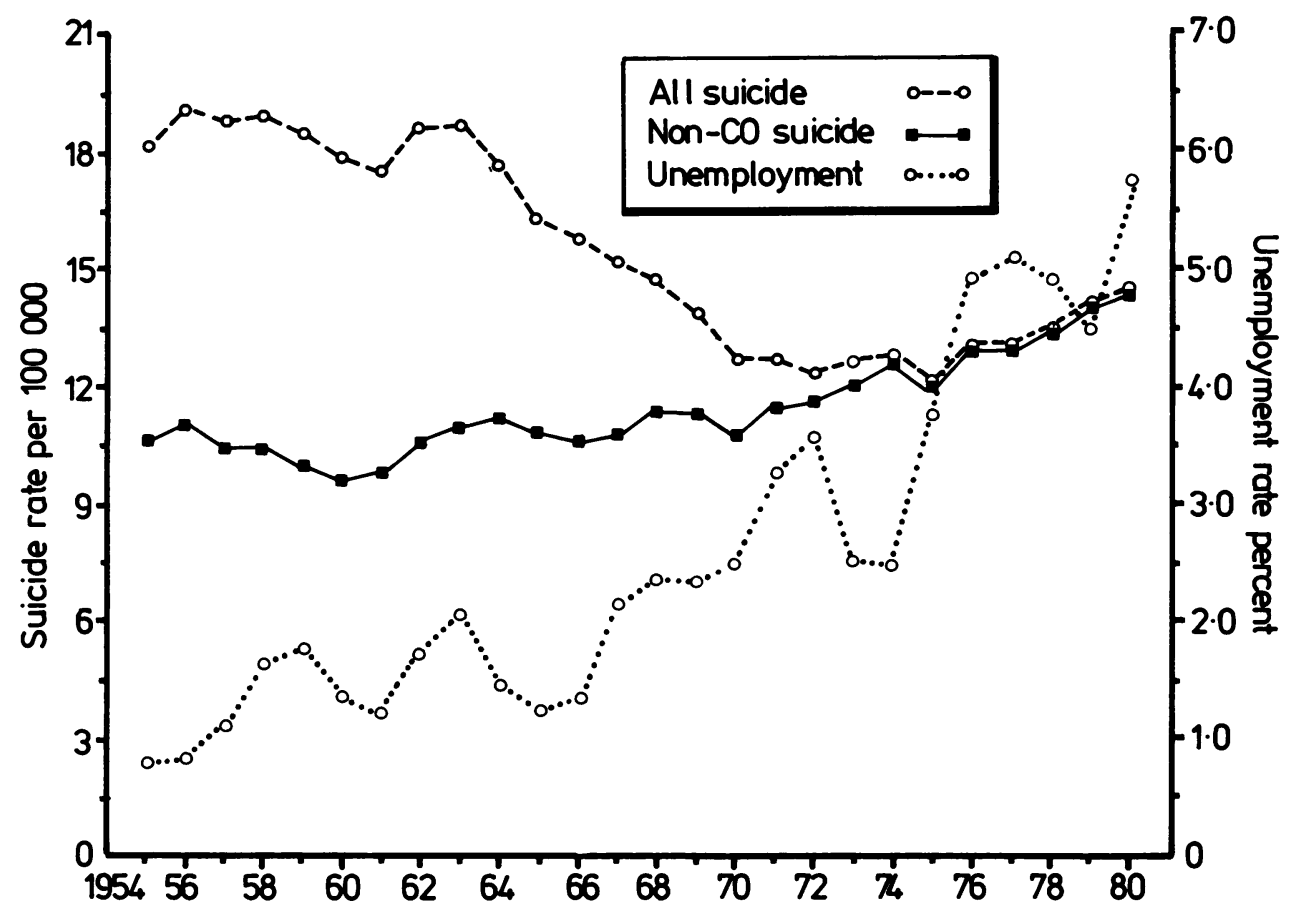

Fig 3 Britain: male suicide and unemployment rates 195580.

age of 65 and others outside the workforce cannot be classified as unemployed. The denominator for suicide rates, however, which again is the total adult population, is exactly comparable with the definition incorporated in the numerator. Such considerations must influence the interpretation of our findings.

SUICIDE AND DOMESTIC GAS DETOXIFICATION Our primary concern has been with the sensitivity of total suicide rates to the availability of toxic domestic gas. To this end the well attested association between unemployment and suicide has been used simply as an analytical device for testing our primary hypothesis. The crucial result concerns the period 1964 to 1972 . For the preceding years, 1955-63, we found no significant associations, conceivably because during this period the fluctuations during the relevant variables were minor. For 1964-72 and 1973-80, however, the results were as predicted. From the arguments set out in the introduction we conclude that availability of a particular method of suicide does indeed effect the total suicide rate (and that the British anomaly is thereby explained).

This conclusion, as noted in our earlier paper, ${ }^{1}$ does not fit easily into the traditional thinking on the epidemiology of suicide; most studies have been principally focused on the role of psychiatric morbidity, or alternatively on a variety of social factors. It would appear that an additional variable - namely, availability of method-may now have to be added. There is some collateral support for this contention. Thus in the United States it has been shown that those states with comparitively stricter gun control laws have persistently lower suicide rates due to lower rates of suicide by firearms and despite a minor "compensatory" increase in rates for other types of suicide. ${ }^{89}$ In the United Kingdom poisoning rather than firearms provide the principal means of suicide. It has been argued from a detailed review of a sample of English suicidal deaths that less prescribing of barbiturates might help reduce suicide, ${ }^{10}$ a suggestion in line with findings from Australia. ${ }^{11}$ Johns showed that in England and Wales between 1969 and 1974 a pronounced decline in the prescribing of barbiturates was associated with a decrease in the death rates from poisoning with these drugs (most of which are suicides) ${ }^{12}$; however, the death rates from "all solids and liquids" showed little change over this period, suggesting that compensatory shifts might occur between closely related methods of suicide for example, poisoning by different medications. Substitution within the major modes of suicide, as by the use of one drug rather than another, may be psychologically easier than 
shifts between modes, such as $\mathrm{CO}$ poisoning, violent methods, etc. Space precludes discussion of the possible psychological meaning for the individual of a given mode of suicide, and of the practical possibilities of controlling the availability of methods as a means of suicide prevention.

\section{SUICIDE AND UNEMPLOYMENT}

The relationship between unemployment and suicidal behaviour is complex. ${ }^{15}$ Here we wish only to point out that the correlations reported in this paper are essentially ecological in character. That male unemployment was significantly associated with female suicide between 1955 and 1980 suggests either that male unemployment is correlated with female unemployment, or that it acts as a marker for more diffuse disturbances within the family or the broader social fabric. Furthermore, we have emphasised that our correlations relate event data (for unemployment) to person data (for suicide) thus excluding the very basis on which an argument for direct causality could be based. We are also aware that we have taken no account of the fact that unemployment is more widespread among younger men whereas suicide is more common in the second half of life. There have also been important changes between 1955 and 1980 affecting both our main variables; unemployment has not only risen but has in recent times been of a longer term character than formerly, and the age specific suicide rates for men have also been changing with an increase in younger groups and a decrease in older people.

These are all cogent reasons against using present results to argue for a causal relationship in the sense of changes in suicide rates being attributable to suicide among the unemployed. It is equally possible that the unemployment rate reflects more widespread distress, with a loosening of social cohesion along the lines proposed by Durkheim. ${ }^{13}$ Nevertheless, it is known that the unemployed are considerably overrepresented among male suicides. ${ }^{14}$ Thus the trends in suicide rates over the past decade may be in part due to the increasing numbers of unemployed, though the suicide rate within the unemployed is also likely to have fluctuated substantially according to the character and determinants of unemployment at different times. To explore these matters further it would be necessary to establish the temporal trends of suicide among unemployed individuals, a task which we have not attempted in the present study.

\section{References}

${ }^{1}$ Kreitman N. The coal-gas story. Br J Prev Soc Med 1976; 30: 86-93.

${ }^{2}$ Farmer RDT. Suicide by different methods. Postgrad Med J 1979; 55: 775-9.

${ }^{3}$ Hassall C, Trethowan W. Suicide in Birmingham. Br Med $J$ 1972; i: 717-8.

4 World Health Organisation, Changing patterns in suicide behaviour. EURO report and studies 74. Copenhagen: WHO, 1982.

${ }^{5}$ Sainsbury P, Jenkins J, Levey A. The social correlates of suicide in Europe.In: Farmer R, Hirsh S. eds. The suicide syndrome. London: Croom Helm, London: 1980.

${ }^{6}$ Boor M, Relationships between unemployment rates and suicide rates in eight countries 1962-1976. Psychol Rep 1980; 47: 1095-1101.

${ }^{7}$ Swinscow D. Some suicide statistics. BR Med J 1951; i: 1417-23.

${ }^{8}$ Lester D, Murrell M. The influence of gun control laws on suicidal behaviour. Am J Psychiatry 1980; 137: 121-2.

${ }^{\circ}$ Lester D, Murrell M. The preventive effect of strict gun control laws on suicide and homicide. Suicide and Life Threatening Behaviour 1982; 12: 131-40.

${ }^{10}$ Barraclough B, Nelson B, Bunch J, Sainsbury P. Suicide and barbiturate prescribing. J R Coll Gen Pract 1971; 21: 645-53.

${ }^{11}$ Oliver RG. Hetzel BS. An analysis of recent trends in suicide rates in Australia. Int J Epidemiol 1973; 2: 1973; 2: 91-101.

12 Johns M. Self-poisoning with barbiturates in England and Wales during 1959-74. Br Med J 1977; i: 1128-30.

${ }^{13}$ Durkheim E. Le suicide. Paris: 1897.

${ }^{14}$ Shepherd D, Barraclough B. Work and suicide: an empirical investigation. Br J Psychiat 1980; 136: 46978.

${ }^{15}$ Platt $S$. Unemployment and suicidal behaviour: a review of the literature. Soc Sci Med (in press). 